\title{
INSTRUMENTO DE AVALIAÇÃO DO REPERTÓRIO BÁSICO PARA ALFABETIZAÇÃO (IAR): aplicabilidade para crianças com transtorno do espectro autista
}

\author{
Najra Danny Pereira Lima? \\ Cássia Leal da Hora²
}

\section{RESUMO}

Avaliação inicial de repertório é uma ferramenta importante para o planejamento de ensino e pode auxiliar na inclusão escolar de indivíduos com Transtorno do Espectro Autista. O IAR foi desenvolvido para avaliar repertório comportamental, pré-requisito para alfabetização de crianças com desenvolvimento neurotípicos. Este instrumento de avaliação é considerado uma ferramenta de avaliação eficaz, simples e de rápida e fácil aplicação. O objetivo do presente trabalho foi verificar a aplicabilidade do IAR em crianças com TEA. Participaram três meninos diagnosticados com TEA, com idades entre 8 e 9 anos, alfabetizados e matriculados no $2^{\circ}$ e $3^{\circ}$ ano fundamental do ensino regular. A aplicação do instrumento e a interpretação dos resultados foram realizadas a partir das instruções fornecidas no manual de aplicação do IAR. Ao final da avaliação, Pl apresentou desempenho indicativo de 'muita dificuldade' na maioria das áreas do instrumento (8 do total de 13). P2 demonstrou 'muita dificuldade' ou 'alguma dificuldade' em 10 áreas e P3 apresentou "alguma dificuldade" em 8. Esses resultados, aliados a outras medidas de pouco engajamento na tarefa, comportamentos disruptivos e padrões de erro, indicam que o formato original da avaliação pode dificultar a realização das tarefas por alunos com TEA. Futuros estudos deveriam investigar se adaptações ao instrumento poderiam torná-lo aplicável a alunos com o diagnóstico de TEA.

Palavras-chave: Avaliação. Pré-requisitos para alfabetização. IAR. Transtorno do Espectro Autista.

\section{INSTRUMENT TO ASSESS OF BASIC REPERTOIRE FOR LITERACY (IAR): applicability}

\section{for children with autism spectrum disorder}

\begin{abstract}
Initial repertoire evaluation is an important tool for teaching planning, and it may assist in the school inclusion of individuals with Autism Spectrum Disorder (ASD). The

\footnotetext{
1 Mestre Profissional em Análise do Comportamento Aplicada (Paradigma - Centro de Ciências e Tecnologia do Comportamento). Orcid iD: 000-0003-3673-0876. E-mail: najradannypl@gmail.com

2 Doutora em Psicologia Experimental - PUC-SP. Docente permanente do Programa Ciências e Tecnologia do Comportamento. Orcid iD: 0000-0001-5029-9600. E-mail:
} Mestrado Profissional em Análise do Comportamento Aplicada no Paradigma - Centro de cassiadahora@paradigmaac.org
\end{abstract}


Instrument to Assess Basic Repertoire for Literacy (IAR) was developed to assess prerequisite behavioral repertoire for the literacy of children with neurotypical development. This evaluation instrument is considered an effective, simple, quick and intuitive assessment tool. The present study aims to verify the applicability of the IAR in children with ASD. Three boys diagnosed with ASD, aged between 8 and 9 years, literate and enrolled in the 2 nd and 3rd elementary years of regular education participated in the study. The application of the instrument and the interpretation of the results were realized based on the instructions provided in the IAR application manual. At the end of the evaluation, $\mathrm{Pl}$ showed indicative performance of 'A lot of difficulty' in most areas of the instrument (8 out of 13). P2 showed 'A lot of difficulty' or 'Some difficulty' in 10 areas and P3 showed 'Some difficulty' in 8 areas. These results combined with other indications of little engagement in the task, disruptive behavior and error patterns, indicate that the original format of the assessment can make it difficult for students with ASD to perform tasks. Future studies should investigate whether adaptations to the instrument could make it applicable to students diagnosed with ASD.

Keywords: Assessment. Literacy Prerequisites. IAR. Autism Spectrum Disorder.

\section{INSTRUMENTO BÁSICO DE EVALUACIÓN DEL REPERTORIO PARA LA ALFABETIZACIÓN (IAR): aplicabilidad para niños con trastorno del espectro autista}

\section{RESUMEN}

La evaluación inicial del repertorio es una herramienta importante para la planificación de la enseñanza y puede ayudar en la inclusión escolar de personas com Trastorno del Espectro Autista (TEA). El Instrumento para la Evaluación del Repertorio de Alfabetización Básica (IAR) fue desarrollado para evaluar el repertorio conductual prerrequisito para la alfabetización de niños con desarrollo neurotípico. Este instrumento de evaluación es considerado una herramienta de evaluación eficaz, simple, rápida y fácil de usar. El objetivo del presente estudio fue verificar la aplicabilidad del IAR en niños con TEA. Participaron del estúdio tres niños diagnosticados con TEA, de entre 8 y 9 años, alfabetizados y matriculados en $2^{\circ}$ y $3^{\circ}$ años de la educación primaria regular. La aplicación del instrumento y la interpretación de los resultados se realizaron con base en las instrucciones proporcionadas en el manual de aplicación del IAR. Al final de la evaluación, el PI mostró un desempeño indicativo de "mucha dificultad" en la mayoría de las áreas del instrumento (8 de 13). P2 mostró 'mucha dificultad' o 'algo de dificultad' en 10 áreas y P3 mostró 'algo de dificultad' en 8 áreas. Estos resultados, combinados con otros indicativos de poca participación en la tarea, conductas disruptivas y patrones de error, indican que el formato original de la evaluación puede dificultar que los estudiantes con TEA realicen tareas. Los estudios futuros deben investigar si adaptaciones al instrumento podrían hacerlo aplicable a estudiantes diagnosticados con TEA.

Palabras clave: Evaluación. Pre-requisitos para la alfabetización. IAR. Trastorno del Espectro Autista. 


\section{INTRODUÇÃO}

O Transtorno do Espectro Autista (TEA) é caracterizado pela manifestação de deficiências na comunicação, nas habilidades de socialização e na existência de padrões restritos e repetitivos de comportamentos, interesses e atividades (American Psychiatric Association [APA], 2013). O repertório comportamental comumente exibido por alunos com TEA pode apresentar comportamentos motores ou verbais estereotipados; respostas incomuns aos inputs sensoriais do ambiente ou excessiva aderência à rotina. Estes déficits e excessos comportamentais podem ser barreiras de aprendizagem em contextos educacionais tradicionais, caso não haja um direcionamento eficaz (GOMES; MENDES, 2010).

Papim e Sanches (2013), apontam que o professor tem um sentimento de angústia diante das alterações comportamentais de alunos com TEA, da pouca responsividade do aluno às investidas pedagógicas e das exigências por desempenho pelo sistema de ensino. Segundo os autores, para direcionar o atendimento prestado pelo professor é necessária a adoção de uma prática estruturada, sistematizada, que inclua avaliação inicial do repertório existente para então realizar um planejamento com procedimentos de ensino que respeitem o estilo de aprendizagem do aluno.

Apesar das características comportamentais comuns no repertório de alunos com TEA, ao que parece, a escola regular ainda mantém a tradição de focar sua atenção em atender necessidades educacionais comuns, delineando objetivos sem avaliar o repertório de entrada de cada aluno. Essa postura tradicional, no âmbito curricular, é demonstrada por propostas rígidas e homogeneizadoras, que desconsideram a dinâmica flexível dos processos de ensino e aprendizagem (BLANCO, 2004). Diante disto, há evidências de que estudantes com TEA participam pouco das atividades da escola, que a interação com os colegas é escassa e que a aprendizagem de conteúdos pedagógicos é limitada (GOMES; MENDES, 2010).

Para o desenvolvimento de programas de ensino eficazes é essencial a realização de uma avaliação repertório de base do aluno que seja 
abrangente e eficiente (SKINNER,1979). Avaliações desse tipo, permitem ao educador conhecer o repertório de entrada do aluno e a definir o ponto de partida que norteará a elaboração do planejamento de ensino de forma a favorecer a prática pedagógica (DE ROSE, 2004).

Existem diversos instrumentos comportamentais para avaliação de diferentes domínios do repertório de indivíduos com TEA, por exemplo, VBMAPP 3 , ABLLS-R ${ }^{4}$, PEP-R 5 e AFLS 6 . Entretanto, eles são comumente utilizados para delinear intervenções comportamentais em contextos terapêuticos e individuais. Para aplicação, é necessário ter familiaridade com a leitura na língua inglesa (ou ter acesso a traduções não validadas), providenciar uma grande quantidade de materiais, utilizar métodos de avaliação direta e indireta, entrevistar diversos interlocutores como familiares e outros profissionais envolvidos na intervenção.

Além disso, essas avaliações costumam requerer um longo tempo de aplicação (podendo levar, dias, semanas ou até meses) e, sobretudo, conhecimentos prévios específicos sobre o manuseio do instrumento ou sobre princípios teóricos que os embasam. Tais princípios teóricos não necessariamente fazem parte da formação dos professores da Educação Básica (exemplo, a visão skinneriana sobre comportamento verbal, para o Uso do VBMAPP). Ainda menos provável o acesso às especificidades sobre a utilização e formas de interpretação dos instrumentos comumente utilizados com a população de TEA. Portanto, tais instrumentos não parecem ser uma alternativa viável para auxiliar os professores da rede regular de ensino a avaliarem seus alunos com TEA e posteriormente, delinear programas educacionais eficazes.

Neste cenário, buscou-se na literatura brasileira alguma ferramenta de avaliação que tivesse demonstrado eficácia em sua aplicação e cujo conteúdo fosse mais compatível com a formação de professores da

\footnotetext{
3 Verbal Behavior Milestones Assessment and Placement Program (SUNDBERG, 2008).

${ }_{4}^{4}$ Assessment of Basic Language and Learning Skills Revised (PARTINGTON, 2006).

5 Psychoeducational Profile Revised (SCHOPLER, REICHLER, BASHFOD, LANSING, \& MARCUS, 1990).

${ }^{6}$ Assessment of Functional Living Skills (PARTINGTON, 2013).
} 
Educação Básica no Brasil que poderiam se apoiar para auxiliar alunos com TEA a responderem suas demandas no contexto educacional.

\section{IAR: Instrumento de Avaliação de Repertório Básico para Alfabetização}

$\mathrm{Na}$ tentativa de fornecer um instrumento que pudesse auxiliar professores da educação básica da rede pública a identificar o repertório de entrada de alunos, e subsidiar a elaboração de estratégias de ensino que favorecessem o desenvolvimento de repertórios envolvidos no processo de alfabetização destes alunos, Leite, em 2013, publicou o IAR, Instrumento de Avaliação de Repertório Básico para Alfabetização. A ferramenta surgiu como uma possibilidade (e ainda o é), para avaliar o repertório comportamental de crianças neurotípicas com faixa etária pré-escolar (5-6 anos), do primeiro ano do ensino fundamental.

Trata-se de um instrumento diante do qual o aluno realiza uma série de tarefas que avaliam habilidades referentes a 13 áreas, consideradas prérequisitos para desenvolver leitura e escrita, a saber: Esquema corporal, Lateralidade, Posição, Direção, Espaço, Tamanho, Quantidade, Forma, Discriminação visual, Discriminação auditiva, Verbalização de palavras, Análise-síntese, Coordenação motora fina (LEITE, 2013). As áreas e tarefas que compõem o instrumento de avaliação foram selecionadas a partir de uma pesquisa na literatura (POPPOVIC et al., 1977), e avaliam habilidades que representam o que comumente se considerou a partir da revisão como pré-requisito para alfabetização na área da educação.

Segundo o manual do instrumento, a duração prevista para finalizar a aplicação do IAR é equivalente ao tempo de uma aula (o que corresponde a aproximadamente 1 hora). Portanto, pode ser considerada como rápida. A aplicação pode ser realizada em sala de aula, em contexto coletivo com um grupo de 10 a 15 alunos (permitindo assim que o professor tenha uma visão global de todos os alunos de sua turma, em relativamente pouco tempo).

O kit de aplicação é composto por um caderno de respostas (que contém as tarefas da avaliação) e um manual para o professor (contém as 
informações que o professor precisará para aplicar o instrumento). As instruções para aplicação são simples, não requerem conhecimentos prévios sobre abordagens teóricas específicas ou necessidades educacionais especiais e podem ser facilmente implementadas pelo professor. Os materiais necessários para a realização das tarefas pelo aluno são: um lápis de cor nas cores verde, azul e amarelo, um lápis grafite e uma borracha.

Após a finalização da avaliação o desempenho do aluno é plotado em uma folha de registro única exibindo os acertos e erros emitidos nas tarefas de todas as áreas. A partir de uma análise simples, o instrumento propõe um sistema de classificação definido como 'Acerto Total', 'Alguma Dificuldade' e "Muita Dificuldade" realizado através do total de respostas em cada uma das 13 áreas do instrumento. Por meio da proposta de direcionamento dos resultados trazida pelo instrumento, o desempenho compatível com 'Acerto Total' indicaria que o aluno demonstra repertório compatível com a habilidade avaliada de forma consistente e, portanto, não haveria indicação de realizar programas educacionais para desenvolvê-los. Os resultados classificados como 'Acerto Total', 'Alguma Dificuldade' indicariam em quais das áreas avaliadas o aluno possui o repertório de forma inconsistente ou não o possui o pré-requisito para alfabetização avaliado. A partir desses resultados, o professor tem informações concretas para realizar a programação de uma ação educacional que objetive a aquisição de habilidades básicas pendentes para promover o processo de alfabetização. Além disso, os professores podem recorrer ao livro "Preparando a Educação", do mesmo autor do IAR, para uma descrição pormenorizada de programas para o desenvolvimento de habilidades básicas nas áreas em que os alunos demonstraram mais dificuldades.

Desde o seu surgimento, diversas experiências de utilização do IAR na rede pública de ensino do Brasil demonstraram a sua eficácia em subsidiar programas de ensino que promovam o aprendizado dos repertórios requisitos para o processo de alfabetização comumente adquiridos em idade préescolar e primeiro ano do ensino fundamental (para maiores informações, 
ver relatos dessas experiências em Leite, 2015). Todavia, apesar do IAR demonstrar sua eficácia e utilidade ao longo dos anos (LEITE, 2015), e de continuar parecendo uma possibilidade em nortear os professores da educação básica, ele foi desenvolvido para avaliar crianças com o neurodesenvolvimento típico, ou seja, sem diagnósticos formais indicativos de deficiências que podem implicar em barreiras de aprendizagem. Fica então o questionamento se a mesma ferramenta poderia ser adotada para auxiliar educadores com interesse em identificar os repertórios de entrada relacionados à alfabetização de um aluno com TEA.

Diante do exposto, o objetivo deste estudo foi verificar a aplicabilidade do IAR em crianças com Transtorno do Espectro Autista. Para tanto, O IAR foi aplicado em crianças com TEA alfabetizadas, para verificar se elas demonstrariam repertórios compatíveis com àqueles que 0 instrumento se propõe avaliar. A hipótese era de que crianças alfabetizadas demonstrassem repertórios compatíveis com a classificação "Acerto Total" as características do diagnóstico de TEA não seriam relevantes para o processo de avaliação da forma como é proposto. Indicando assim, que o instrumento também poderia ser aplicado também para a população em questão.

\section{MÉTODO}

\section{Participantes}

Participaram deste estudo, três meninos, um com 8 anos $(P 1)$ e dois com 9 (P2 e P3), matriculados em escolas da rede de ensino regular do estado do Maranhão-MA. Pl cursava o $2^{\circ}$ ano do ensino fundamental e P2 e P3 cursavam $\circ 3^{\circ}$ ano. Todos eram diagnosticados com TEA e sabiam ler e escrever fluentemente e, portanto, em nível necessário para responder às demandas do primeiro ano do Ensino Fundamental (compatível com que é avaliado pelo IAR).

Para participar da pesquisa, os seguintes critérios de inclusão foram adotados: os participantes deveriam ter diagnóstico de TEA e exibir 
comportamento básico de estudante (definido no presente estudo como ser capaz de iniciar e concluir uma tarefa simples e seguir instruções verbais simples, com e sem objetos, por exemplo, sentar quando solicitado, atender quando chamado pelo nome, selecionar o objeto quando o mesmo fosse nomeado, etc.). Além disso, os participantes deveriam estar matriculados na rede regular de ensino e cursar entre o primeiro e quarto ano do ensino fundamental (séries que certamente englobariam os repertórios avaliados pelo instrumento). Por fim, os participantes também deveriam ter demonstrado os repertórios avaliados pelo IAR latestados por meio de avaliação comportamental - VBMAPP, formalmente realizada antes da participação no estudo, na clínica onde recebiam serviços educacionais).

\section{Local e materiais}

As sessões de aplicação do instrumento foram conduzidas em uma sala de um consultório particular para atendimento educacional. A sala era mobiliada com uma mesa, duas cadeiras, um armário que permanecia fechado durante a aplicação (contendo materiais educacionais gerais, por exemplo, livros, brinquedos etc.) e uma filmadora NIKON D 7000 para registro das sessões.

Foram utilizados todos os materiais requeridos pelo manual de aplicação do IAR: cadernos de aplicação (manual de aplicação e folhas de respostas), dois lápis pretos (um para $\circ$ participante e outro para a pesquisadora), uma borracha e lápis de cor amarelo, vermelho e verde. Todas as sessões de coleta de dados foram filmadas.

\section{Variáveis e Delineamento Experimental}

O estudo foi realizado a partir da lógica dos delineamentos de sujeito único, na qual os mesmos participantes são submetidos a diferentes sessões de mensuração do comportamento alvo investigado e as manipulações experimentais, quando necessárias, são realizadas com base no desempenho do próprio participante (COOPER; HERON; HEWARD, 2007). OU seja, o sujeito é o seu próprio controle na medida em que é comparado, 
sobretudo, com ele mesmo (em oposição aos delineamentos de grupo, nos quais a comparação dos resultados é feira entre os grupos intervenção e controle).

Em um contexto de manipulação experimental, a variável independente poderia ser considerada o instrumento de avaliação IAR e as variáveis dependentes: as respostas corretas às tarefas, o tempo total de aplicação, engajamento na tarefa e emissão de comportamentos disruptivos. Entretanto, os participantes não foram submetidos a uma condição experimental planejada de Linha de Base (caracterizando um delineamento pré experimental do tipo $A B$ ), pois a questão investigada aqui, diz respeito justamente à aplicabilidade do instrumento de avaliação IAR tal qual ele se apresenta no momento. Apresentar o mesmo instrumento duas vezes traria um viés à análise devido à exposição previa às tarefas. Portanto, optou-se por apresentar o IAR somente uma vez, do início ao fim, e mensurar diversas categorias de comportamentos que serão apresentadas a seguir.

\section{Procedimento}

Para a aplicação do IAR foram seguidas as orientações do manual de aplicação, estritamente da forma como aparecem descritas. Assim, antes de iniciar a coleta de dados, a sala foi organizada de modo a reduzir os estímulos ambientais que pudessem servir como distratores para os participantes (por exemplo, itens de decoração ou brinquedos pedagógicos que pudessem distrair a criança). A aplicação foi realizada na modalidade individual (ao invés de aplicação em grupo).

Foram dispostos na mesa o caderno de aplicação do IAR, o lápis preto e a borracha a ser utilizada pelo participante e os materiais para registro a serem utilizados pela pesquisadora. Os demais materiais eram disponibilizados para criança à medida que eram necessários para a realização das tarefas.

As sessões de coletas dos dados ocorreram em horários programados e acordados entre pesquisadora e os responsáveis pela criança. Cada sessão tinha duração de aproximadamente 1 hora cada e à medida em 
que eram necessárias mais sessões para finalização da avaliação, novos horários eram arranjados. As sessões foram realizadas em dias consecutivos. O tempo de aplicação do instrumento de avaliação iniciava a partir do momento em que a experimentadora fornecia instrução para a primeira tarefa (com o participante já sentado à mesa).

Foram apresentados intervalos para descanso do participante sempre que necessário (quando o participante pedisse ou após um breve período em que estava realizando a tarefa após ter demostrado comportamentos indicativos de cansaço).

A sessão ou a aplicação do instrumento era finalizada depois que todas as tarefas eram apresentadas ou quando o tempo disponível para aquela sessão era finalizado e o participante tinha que ir embora. Estavam presentes no momento da coleta somente a pesquisadora e um dos participantes.

A participação de todas as crianças foi autorizada por meio da assinatura do TCLE - Termo de Consentimento Livre e Esclarecido pelos seu respectivos responsáveis e o Projeto foi submetido à Plataforma Brasil e aprovado com o número de processo 2.677.489.

\section{Medidas}

Foram alvo de interesse do presente estudo as seguintes medidas:

(A) Classificação do desempenho por área: A classificação do desempenho dos participantes em cada área foi realizada a partir dos seguintes critérios: Acerto Total $(\mathrm{AT})=100 \%$ de acerto das tarefas da área; Alguma Dificuldade $(A D)=$ acerto entre $50 \%$ e $99 \%$ e Muita Dificuldade $(M D)=$ número de acerto menor que $50 \%$ ( $\leq 49 \%)$ das tarefas da área. Tais critérios são descritos especificamente no manual de aplicação do IAR.

O manual do IAR não descreve as categorias de medidas nos itens $B$ a $F$ descritos a seguir. Tais informações foram coletadas no presente estudo com o objetivo de auxiliar a análise acerca da aplicabilidade do instrumento para crianças com TEA. 
(B) Número e porcentagem de acertos e erros emitidos pelos participantes em cada área avaliada: o cálculo da porcentagem de acertos e erros foi realizado dividindo o número total de vezes em que o participante acertou ou errou a tarefa proposta, pelo número total de oportunidades de responder e multiplicado por 100.

(C) Tempo de duração da aplicação do instrumento: tempo total em minutos para a finalização da avaliação com cada um dos participantes.

(D) Tempo de engajamento nas tarefas da avaliação: descrito aqui como olhar em direção à experimentadora enquanto oferece instrução (pode ser acompanhado de assentimento ou afirmação com a cabeça ou vocalização); escrever no material a resposta solicitada (independente de acerto ou erro); repetir o que foi solicitado para confirmação; responder ao material da avaliação (apontando para, escrevendo sobre ou apontando em). Para registro de engajamento nas tarefas foi utilizado o procedimento de registro descontínuo por intervalo de tempo lamostra de tempo momentânea, 5 min).

(E) Ocorrência de comportamentos disruptivo: Considerou-se como disruptivo qualquer tipo de comportamento que interrompesse a cadeia de aprendizagem e/ou a execução da tarefa. Comportamentos com duração contínua e frequência alta ou moderada foram registrados por meio do sistema de registro de intervalo parcial (1min). Na sessão Resultados/Discussão, serão apresentadas as diferentes categorias de respostas que compuseram essa classe de comportamento.

(F) Tipos de erros emitidos pelos participantes: o presente estudo categorizou os tipos de erro cometidos pelos participantes com o intuito de identificar alguma relação do desempenho obtido e o material apresentado (ainda que o IAR não proponha este tipo de análise). A seguir, aparecem listadas as categorias dos tipos de erros: 
(1) Respostas discrepantes (RD): o participante executou uma ação de forma incorreta quando realizou qualquer ação diferente à requerida na instrução.

(2) Respostas incompletas (RIN): o participante executou somente uma parte do que foi solicitado pela ação descrita na instrução ou deixou de responder alguma das demandas requeridas para completar a tarefa.

(3) Respostas adicionais (RA): o participante executou na folha de respostas a ação requerida na instrução e alguma outra resposta não requerida.

(4) Ausência de resposta (AR): o participante não emitiu nenhuma resposta em relação à instrução requerida.

\section{Acordo entre observadores}

A medida de acordo entre observadores (AEO) sobre a ocorrência de comportamentos disruptivos foi obtida a partir do cálculo do número de intervalos com acordo, dividido pela somatória do número de intervalos com acordo e sem acordo, multiplicado por 100. Para tanto, 100\% das sessões foram analisadas duas outras observadoras independentes treinadas para observar e identificar as respostas alvo. A porcentagem de AEO sobre os comportamentos disruptivos de $\mathrm{P} 1$ foi de $100 \%$; para os de $\mathrm{P} 2$ de $98 \%$ e para os de $\mathrm{P} 3$ de $100 \%$.

\section{RESULTADOS E DISCUSSÃO}

Os resultados serão apresentados em figuras e tabelas considerando individualmente o repertório de cada um dos participantes nas diferentes áreas do IAR, bem como em cada uma das tarefas do instrumento.

Tabela 1. Classificação geral do desempenho de P1, P2 e P3 segundo os critérios do IAR 


\begin{tabular}{llccc}
\hline \multicolumn{1}{c}{ Áreas do IAR } & P1 & P2 & P3 \\
\hline \hline A 1 & Esquema corporal & AT & AT & AD \\
A 2 & Lateralidade & MD & MD & AD \\
A 3 & Posição & MD & MD & AD \\
A 4 Direção & AT & AT & AT \\
A 5 Espaço & MD & MD & AD \\
A 6 & Tamanho & MD & MD & AD \\
A 7 Quantidade & MD & MD & AD \\
A 8 Forma & AT & AD & AT \\
A 9 Discriminação Visual & MD & MD & AD \\
A 10 Discriminação Auditiva & MD & MD & MD \\
A 11 Verbalização De Palavras & MD & AT & AD \\
A 12 & Análise e Síntese & AD & MD & AD \\
A 13 Coordenação Motora Fina & AT & MD & AT \\
\hline
\end{tabular}

Nota. $A T=$ Acerto Total; $A D=$ Alguma Dificuldade; $M D=$ Muita dificuldade

Fonte: as autoras.

A Tabela 1 mostra a classificação geral do desempenho de P1, P2 e P3 nas áreas avaliadas pelo instrumento IAR. Pl obteve desempenho compatível com a classificação 'Acerto Total' em 4 das 14 áreas no total, a saber, Al Esquema Corporal, A4 Direção, A8, Forma e A13 Coordenação Motora Fina. Esses resultados indicam que as habilidades pré-requisito requeridas por essas áreas estavam presentes de forma consistente no repertório de $\mathrm{P} 1$. P2 respondeu com AT somente nas áreas $\mathrm{A} 1$ e A2. Por sua vez, P3 respondeu com AT nas áreas A4, A8 e A13.

Também é possível observar na Tabela lque o desempenho de P1 foi classificado com 'Muita Dificuldade' (MD) na maioria das áreas avaliadas pelo instrumento (8 das áreas), P2 em 9 das 13 áreas e P3 em apenas uma das 13 áreas. Pl teve desempenho compatível com a classificação 'Alguma dificuldade' (AD) na área Análise-Síntese, P2 na área forma, e P3 na maioria das áreas do IAR (9 de 13). Apenas uma das áreas foi classificada com 'Acerto Total' (AT) para todos os três participantes, a A4 (Direção). Por outro lado, todos os participantes exibiram desempenho compatível com MD na A10 (Discriminação Auditiva). A área 'Verbalização de palavras' foi classificada como AT somente a partir do desempenho de P2.

Os resultados da Tabela 1 indicam que, segundo a avaliação do IAR, os três participantes deveriam receber programas de ensino que 
objetivassem desenvolver a maioria das habilidades consideradas como prérequisito para o aprendizado de leitura e escrita e compatíveis com as habilidades necessárias para responder às demandas do $1^{\circ}$ ano do ensino básico, comumente chamadas de pré-requisitos para a alfabetização.

As Tabelas 2, 3 e 4 mostrarão o desempenho dos Participantes P1, P2 e P3, respectivamente. Em cada uma delas, será possível observar o desempenho dos participantes em cada uma das tarefas apresentadas em cada uma das 13 áreas que compõem o instrumento. Além disso, nas tabelas serão apresentados os tipos de erros cometidos por este participante (RA= Resposta Adicional; $R D=$ Resposta Discrepante; $R I N=$ Resposta Incompleta; e AR= Ausência de resposta).

Importante mencionar que cada célula da tabela representa uma tarefa da avaliação (ver primeira coluna). As células em cinza claro representam ACERTOS e em cinza escuro, ERROS. As células em branco significam que não existe a tarefa. As siglas presentes nos erros representam o tipo de erros emitido pelo participante naquela tarefa.

Tabela 2. Porcentagem de acerto e tipos de erros emitidos por Pl nas tarefas de cada área do IAR

\begin{tabular}{|c|c|c|c|c|c|c|c|c|c|c|c|c|c|}
\hline \multicolumn{14}{|c|}{ ÁREAS DO IAR } \\
\hline TAREFAS & A1 & A2 & A3 & A4 & A5 & A6 & A7 & A8 & A9 & A 10 & A11 & A12 & A13 \\
\hline $\mathrm{T} 1$ & & RA & $\mathrm{RD}$ & & $\mathrm{RD}$ & $\mathrm{RD}$ & $\mathrm{RD}$ & & $\mathrm{RD}$ & $\mathrm{AR}$ & RIN & $\mathrm{RD}$ & \\
\hline $\mathrm{T} 2$ & & RIN & $\mathrm{RD}$ & & $\mathrm{RD}$ & $\mathrm{RD}$ & & & $\mathrm{RD}$ & $\mathrm{AR}$ & & & \\
\hline $\mathrm{T} 3$ & & $\mathrm{RD}$ & & & & & $\mathrm{RD}$ & & & $\mathrm{AR}$ & & & \\
\hline $\mathrm{T} 4$ & & AR & $\mathrm{RD}$ & & & $\mathrm{RD}$ & RD & & RA & $\mathrm{AR}$ & & $\mathrm{RD}$ & \\
\hline T5 & & & $\mathrm{RD}$ & & & & & & RA & & & & \\
\hline $\mathrm{T} 6$ & & & $\mathrm{AR}$ & & & $\mathrm{RD}$ & $\mathrm{RD}$ & & RA & & & $\mathrm{AR}$ & \\
\hline $\mathrm{T} 7$ & & & & & & $\mathrm{RD}$ & & & & & & & \\
\hline $\mathrm{T} 8$ & & & & & & $\mathrm{RD}$ & & & & & & & \\
\hline TOTAL & $\begin{array}{c}100 \% \\
(6 / 6)\end{array}$ & $\begin{array}{c}0 \% \\
(0 / 4)\end{array}$ & $\begin{array}{c}16,60 \% \\
(1 / 6)\end{array}$ & $\begin{array}{c}100 \% \\
(2 / 2)\end{array}$ & $\begin{array}{c}0 \% \\
(0 / 2)\end{array}$ & $\begin{array}{l}25 \% \\
(2 / 8)\end{array}$ & $\begin{array}{l}33 \% \\
(2 / 6)\end{array}$ & $\begin{array}{r}100 \% \\
(4 / 4)\end{array}$ & $\begin{array}{c}16,60 \% \\
(1 / 6)\end{array}$ & $\begin{array}{c}0 \% \\
(0 / 4)\end{array}$ & $\begin{array}{l}0 \% \\
(0 / 1)\end{array}$ & $\begin{array}{l}50 \% \\
(3 / 6)\end{array}$ & $\begin{array}{r}100 \% \\
(1 / 1)\end{array}$ \\
\hline
\end{tabular}

Nota. $R A=$ Resposta Adicional; $R D=$ Resposta Discrepante; $R I N=$ Resposta Incompleta; e $A R=$ Ausência de resposta

Fonte: as autoras.

É possível observar na Tabela 2 que $\mathrm{P} 1$ emitiu 22 respostas corretas e 34 incorretas nas 56 oportunidades de responder, isso corresponde a 39,3\% de acerto e $60,7 \%$ de erros no total. Ademais, mesmo sendo alfabetizado, P1 teve desempenho de $100 \%$ de acertos em apenas 4 das 13 áreas do IAR (A), A4, A8 e A13), e 0\% de acerto em, também, 4 áreas (A2, A5, A10 e A1 1). 
Também é possível observar que a maioria dos erros (21 do total de 34, que corresponde à $61,7 \%$ ), foi da categoria 'Respostas Discrepantes' (RD). Esses resultados indicam que quando errava, na maioria das vezes, o Pl executava uma ação diferente da que foi solicitada pela instrução.

Uma análise qualitativa das respostas incorretas mostrou que os dois tipos de erros mais cometidos por P1 (RD e AR, respectivamente) ocorreram, principalmente, nas tarefas que requeriam ações manuscritas, por exemplo, pintar, fazer um X ou circular uma da opções de escolha apresentadas em arranjos de estímulos linearmente (alinhados lado a lado) com no mínimo quatro figuras apresentadas lado a lado. Nestes casos, foi possível constatar que, a partir da primeira tarefa da área A5 (Tamanho) o participante sistematicamente respondeu sobre os dois primeiros estímulos da esquerda (ou somente no primeiro) do arranjo linear (pintando, circulando ou fazendo um $x$ sobre). Este padrão também ocorreu em duas das quatro oportunidades na área A6 (Tamanho), em que as tarefas tinham as mesmas características (4 ou mais figuras dispostas lado a lado em um arranjo linear), em todas as três tarefas que compõem a área A7 (Quantidade), em três das sete oportunidades da área A9 (Discriminação Visual) e, por fim, em quatro das oito possibilidades na área A10 (Discriminação Auditiva).

Outros padrões semelhantes foram observados no mesmo tipo de tarefa da área A10. Um deles, foi a reprodução das respostas de circular os estímulos nas mesmas posições em que o instrumento apresentava o modelo da tarefa (nas quais o professor deve oferecer modelo de como realizá-la, a partir de uma demanda de "Exemplo"). Existiam ainda outras demandas em que havia um quadrado na porção esquerda da página, contendo uma figura com função de estímulo condicional que deveria evocar a resposta correta. Os dois tipos de tarefa supramencionados, mesclaram demandas com e sem a apresentação do "exemplo" e com e sem o quadrado com a figura "modelo" ao lado esquerdo. Ainda na área A10, havia o total de 8 tarefas, dos dois tipos (com e sem modelo ou com e sem Exemplo). Em todas elas, P1 fez um círculo na figura disposta na primeira localização à esquerda do arranjo linear de quatro estímulos (figuras). 
Desses resultados decorrem duas hipóteses: (a) o participante pode ter acertado ao acaso algumas das tarefas que compõem as áreas A5, A6, A7, A9 e A10; e (b) a disposição visual dos estímulos na página, além da variabilidade de tarefas com e sem "Exemplo" ou com e sem "modelo", pode ter favorecido a ocorrência de respostas indicativas de que o participante respondeu sob controle da localização dos estímulos em que estes eram apresentados na página. Esses resultados corroboram com alguns estudos que apontam para o risco do estabelecimento deste desempenho em crianças com TEA submetidas a tarefas de discriminação condicional com o procedimento de escolha de acordo com o modelo (DA HORA, DEBERT, LAFRANCE \& MIGUEL, 2018).

De forma geral, os resultados indicam que P1 não conseguiu realizar a maior parte das tarefas propostas pelo IAR no formato em que são apresentadas. Segundo os resultados obtidos a partir do IAR e da sua proposta de classificação e interpretação dos mesmo, caso seu repertório pré- requisito para a alfabetização tivesse que ser definido com isso, um professor deveria planejar atividades para $\circ$ desenvolvimento das habilidades avaliadas pela maioria das áreas que o instrumento se propõe a avaliar. Ainda que P1 já seja alfabetizado e que já tenha demonstrados as habilidades mensuradas no IAR em outras circunstâncias.

Tabela 3. Porcentagem de acertos e tipos de erros emitidos por P2 nas tarefas de cada área do IAR

\begin{tabular}{|c|c|c|c|c|c|c|c|c|c|c|c|c|c|}
\hline \multicolumn{14}{|c|}{ ÁREAS DO IAR } \\
\hline TAREFAS & A1 & A2 & A3 & A4 & A5 & A6 & A7 & A8 & A9 & A 10 & A11 & A12 & A13 \\
\hline $\mathrm{T} 1$ & & RA & RA & & RD & RD & RD & RA & RD & RA & & RD & RA \\
\hline $\mathrm{T} 2$ & & RA & RA & & RD & & RD & & RA & RA & & RA & \\
\hline $\mathrm{T} 3$ & & RA & RA & & & & & & RA & $\mathrm{RD}$ & & & \\
\hline $\mathrm{T} 4$ & & RA & RA & & & & RA & & & RA & & RA & \\
\hline $\mathrm{T} 5$ & & & $\mathrm{RD}$ & & & RD & & & & & & & \\
\hline $\mathrm{T} 6$ & & & $\mathrm{RD}$ & & & RD & RA & & $\mathrm{RD}$ & & & $\mathrm{RD}$ & \\
\hline $\mathrm{T} 7$ & & & & & & & & & & & & & \\
\hline $\mathrm{T} 8$ & & & & & & RD & & & & & & & \\
\hline TOTAL & $\begin{array}{c}100 \% \\
(6 / 6)\end{array}$ & $\begin{array}{c}0 \% \\
(0 / 4)\end{array}$ & $\begin{array}{c}0 \% \\
(0 / 6)\end{array}$ & $\begin{array}{l}100 \% \\
(2 / 2)\end{array}$ & $\begin{array}{c}0 \% \\
(0 / 2)\end{array}$ & $\begin{array}{l}38 \% \\
(3 / 8)\end{array}$ & $\begin{array}{c}33,0 \% \\
(2 / 6)\end{array}$ & $\begin{array}{l}76 \% \\
(3 / 4)\end{array}$ & $\begin{array}{c}33,0 \% \\
(2 / 6)\end{array}$ & $\begin{array}{c}0 \% \\
(0 / 4)\end{array}$ & $\begin{array}{r}100 \% \\
(1 / 1)\end{array}$ & $\begin{array}{l}33 \% \\
(2 / 6)\end{array}$ & $\begin{array}{c}0 \% \\
(0 / 1)\end{array}$ \\
\hline
\end{tabular}

Nota: RA = Resposta Adicional; RD= Resposta Discrepante; RIN=Resposta Incompleta; e $A R=$ Ausência de resposta).

Fonte: as autoras. 
Na Tabela 3 é possível observar que P2 emitiu apenas 21 respostas corretas nas 56 oportunidades de responder, o equivalente à $37 \%$ de acertos e $63 \%$ de erros no total. Além disso, teve desempenho de $100 \%$ de acertos em apenas 3 das 13 áreas que compõem o instrumento (a saber Al, A4 e A1 1) e $0 \%$ de acerto em 5 dessas áreas (A2, A3, A5, A10 e A13).

Diferente do desempenho de $\mathrm{Pl}$, a Tabela 3 mostra que a maior parte dos erros cometidos por P2 (19 de 35, correspondente à 54,2\%) foram da categoria 'Respostas Adicionais' (RA) indicando que o participante errava a tarefa, pois realizava mais ações do que a instrução solicitava na tarefa.

Os erros adicionais cometidos por P2, consistiam, geralmente, em riscar sobre ou pintar mais do que um estímulo para responder na mesma tarefa ou ainda pintar as figuras das outras tarefas apresentadas na mesma página, uma vez que mais de uma tarefa é apresentada na mesma página do Caderno de Respostas do aluno avaliado.

Embora o tipo de erro mais frequente no desempenho de P2 tenha sido diferente do de $\mathrm{Pl}$, o resultado da avaliação também nortearia a conduta da professora de P2 de forma similar. Considerando que P2 não conseguiu realizar com acerto pelo menos metade das tarefas propostas pelo IAR, e a partir das diretrizes de classificação e interpretação dos resultados do instrumento, mesmo sendo alfabetizado, assim como P1, P2 também seria candidato a realização de atividades para o desenvolvimento das habilidades avaliadas por diversas tarefas e áreas que o IAR se propõe a avaliar.

Tabela 4. Porcentagem de acertos e tipos de erros emitidos por P3 nas tarefas de cada área do IAR

\begin{tabular}{|c|c|c|c|c|c|c|c|c|c|c|c|c|c|}
\hline \multicolumn{14}{|c|}{ ÁREAS DO IAR } \\
\hline TAREFAS & $\mathrm{A} 1$ & $\mathrm{~A} 2$ & A3 & A4 & A5 & A6 & A7 & A8 & A9 & A 10 & A11 & A12 & A 13 \\
\hline $\mathrm{T} 1$ & RIN & & & & & RD & & & RD & & RIN & & \\
\hline $\mathrm{T} 2$ & & & & & RIN & RD & & & & RIN & & & \\
\hline $\mathrm{T} 3$ & & RD & & & & & RD & & & $\mathrm{RD}$ & & & \\
\hline $\mathrm{T} 4$ & & RD & & & & & & & & $\mathrm{RD}$ & & & \\
\hline T5 & & & RD & & & & & & & & & & \\
\hline T6 & & & RD & & & $\mathrm{RD}$ & & & & & & $\mathrm{RD}$ & \\
\hline $\mathrm{T} 7$ & & & & & & & & & & & & & \\
\hline $\mathrm{T} 8$ & & & & & & & & & & & & & \\
\hline TOTAL & $\begin{array}{l}83 \% \\
(5 / 6)\end{array}$ & $\begin{array}{l}50 \% \\
(2 / 4)\end{array}$ & $\begin{array}{l}67 \% \\
(4 / 6)\end{array}$ & $\begin{array}{c}100 \% \\
(2 / 2)\end{array}$ & $\begin{array}{l}50 \% \\
(1 / 2)\end{array}$ & $\begin{array}{l}63 \% \\
(5 / 8)\end{array}$ & $\begin{array}{l}83 \% \\
(5 / 6)\end{array}$ & $\begin{array}{l}100 \% \\
(4 / 4)\end{array}$ & $\begin{array}{l}83 \% \\
(5 / 6)\end{array}$ & $\begin{array}{l}25 \% \\
(1 / 4)\end{array}$ & $\begin{array}{c}0 \% \\
(0 / 1)\end{array}$ & $\begin{array}{l}83 \% \\
(5 / 6)\end{array}$ & $\begin{array}{l}100 \% \\
(1 / 1)\end{array}$ \\
\hline
\end{tabular}


Nota. $R A=$ Resposta Adicional; $R D=$ Resposta Discrepante; $R I N=$ Resposta Incompleta; e $A R=$ Ausência de resposta

Fonte: as autoras.

Pela Tabela 4 observa-se que P3 emitiu 40 respostas corretas e 16 erradas nas 56 oportunidades de responder, correspondendo a $71,4 \%$ de acertos e $28,6 \%$ de erros. Embora P3 tenha sido o único dos três participantes do estudo que obteve porcentagem de acerto maior do que a porcentagem de erros, seu desempenho foi de $100 \%$ de acertos em apenas 3 das 13 áreas do IAR (A4, A8 e A13). P3 obteve $0 \%$ de acerto em apenas uma das áreas, A1 1 (Verbalização de palavras). Também é possível observar que a maioria dos erros (12 de 21 , ou seja, $57 \%$ ) foi da categoria 'Respostas Discrepantes' (RD). Esses resultados indicam que assim como Pl, quando errava, P3 executava uma ação diferente da que foi solicitada pela instrução.

A maioria dos erros emitidos por P3 foram em tarefas nas quais a resposta requeria controle discriminativo por aspectos sutis dos estímulos apresentados. Por exemplo, na tarefa da área Quantidade, na qual o participante precisava identificar o conceito de 'mais ou menos' a partir da quantidade de líquido presente em um arranjo composto por 4 copos com líquido dentro, sendo que a diferença entre as quantidades de água das figuras era bastante sutil. Esses resultados indicam que a diferença crítica entre os estímulos ter sido pouco saliente, pode ter favorecido a ocorrência de erros.

Considerando que o desempenho de P3 foi classificado com a categoria 'Alguma Dificuldade' na grande maioria das tarefas, o resultado final do IAR poderia ser considerado por um possível professor de P3 que o aluno ainda não possuía as habilidades avaliadas de forma consistente. Dessa forma, segundo o sistema de classificação do IAR, P3 também deveria realizar programas para o desenvolvimento de diversas habilidades já atestadas como presentes em seu repertório.

Tabela 5. Tempo de duração da aplicação do IAR com P1, P2 e P3 


\begin{tabular}{lccc}
\hline & P1 & P2 & P3 \\
\hline \hline Número de sessões & 2 & 1 & 1 \\
Duração total em minutos & 93 & 50 & 43 \\
\hline
\end{tabular}

Fonte: as autoras.

A Tabela 5 mostra o tempo total da aplicação do IAR com cada um dos participantes do estudo. Foram necessárias duas sessões para que Pl finalizasse a aplicação do instrumento que teve duração total de 93 minutos (Sessão 1 com 50min e Sessão 2 com 43min). P2 e P3 finalizaram a aplicação em apenas uma sessão cada, com duração total de 59 e 35 minutos, respectivamente. Esses resultados indicam que para dois dos três participantes do estudo, o tempo total de aplicação do instrumento foi semelhante ao tempo proposto pelo manual do IAR para a aplicação com crianças neurotípicas, aproximadamente, uma aula (que costuma durar 50min) (LEITE, 2015). Somente P1, realizou a aplicação em um tempo muito superior (quase o dobro) do tempo recomendado para crianças neurotípicas.

Figura 1. Porcentagem de intervalos com emissão de comportamentos disruptivos por P1, P2 e P3 durante a aplicação das tarefas do IAR

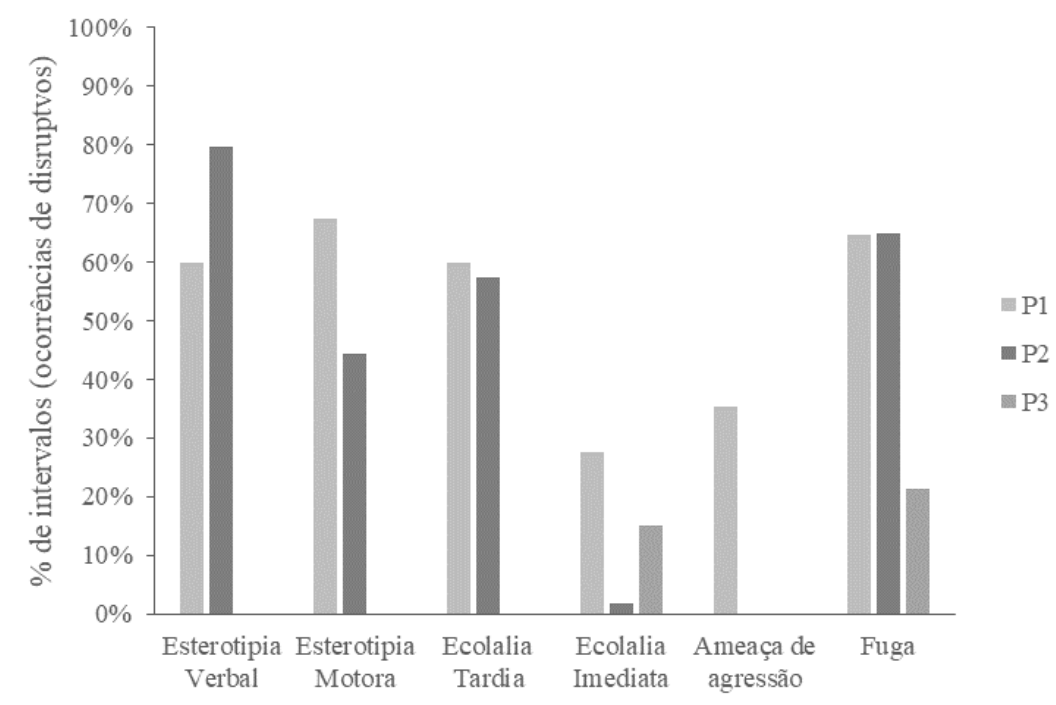

Fonte: as autoras.

A Figura 1 mostra a porcentagem de intervalos nos quais os participantes se engajaram em cada uma das categorias de respostas que 
compuseram a classe de comportamentos disruptivos ao longo da aplicação do IAR. É possível observar que os comportamentos disruptivos mais emitidos por Pl foram os categorizados como estereotipias vocais $160 \%$ dos intervalos), estereotipias motoras (em $70 \%$ dos intervalos), fuga da tarefa (em cerca de 65\%) e ecolalia tardia (em 58\%). Respostas pertencentes a todas essas categorias de disruptivos estiveram presentes em mais de $50 \%$ dos intervalos de aplicação da avaliação. Os comportamentos categorizados como ecolalia imediata e ameaça de agressão também foram emitidos por Pl, mas estiveram presentes por uma quantidade de tempo menor, cerca de $30 \%$ e $35 \%$ dos intervalos registrados, respectivamente.

É válido destacar que os comportamentos chamados de ameaça de agressão (direcionar a mão em direção à boca da experimentadora; direcionar a boca aberta para o próprio braço enquanto falava que ia morder; empurrar ou puxar a mão da experimentadora), eram comportamentos que não ocorriam nas interações com este participante, independente dos contextos de atividade. Isso levanta a hipótese de que o tipo de tarefa apresentado na avaliação pode estar relacionado com o engajamento em comportamentos disruptivos.

A Figura 1 também mostra que os comportamentos disruptivos mais emitidos por P2 foram os categorizados como estereotipias vocais (em aproximadamente $80 \%$ dos intervalos); fuga da tarefa (em aproximadamente $65 \%$ dos intervalos); ecolalia tardia (em aproximadamente $65 \%$ dos intervalos). Assim como no caso de $\mathrm{Pl}$, tais comportamentos disruptivos estiveram presentes em mais da metade do período em que a avaliação foi aplicada. Os comportamentos categorizados como estereotipias motoras e ecolalia imediata também ocorreram, mas estiveram presentes em cerca de $45 \%$ e $2 \%$ dos intervalos, respectivamente.

P3 também emitiu os comportamentos disruptivos categorizados como Ecolalia imediata (em aproximadamente, $15 \%$ dos intervalos) e fuga da tarefa (em aproximadamente $21 \%$ dos intervalos). Em contrapartida, não o 
participante não se engajou em nenhum outro tipo de categoria de comportamento disruptivo. Ao contrário de $\mathrm{P} 1$ e de $\mathrm{P} 2$, comportamentos disruptivos não estiveram presentes na maior parte do tempo de aplicação do instrumento ao P3.

Conforme mencionado anteriormente, a classe de respostas definida como "Comportamentos Disruptivos' considerou aqueles que de alguma forma competiam com a realização da tarefa. Diversas topografias de resposta foram observadas: (e.g., tosses; sons ininteligíveis, inserir o dedo polegar ou indicador no orifício nasal; apertar continuamente a orelha; balançar ou bater, ecolalia imediata e tardia; , direcionar a mão com força em direção à boca da experimentadora; direcionar a boca aberta para o próprio braço enquanto fala, olhando para a pesquisadora: "Eu posso me morder?" Ou "Eu vou me morder!" (sic); empurrar a mão ou puxar a mão da pesquisadora em sua direção ou em direção a tarefa; deixar a área de trabalho e permanecer em pé, ou afastar-se da mesa; direcionar o olhar para a direita ou esquerda após a pesquisadora oferecer a instrução para realizar uma tarefa).

Embora se saiba que comportamentos estereotipados podem ter função comunicativa, indicando ao interlocutor que, ao emiti-los, o aluno poderia estar comunicando que não quer realizar a tarefa, ou que ela está muito difícil (e.g. fuga/esquiva de demandas). Ainda, que o aluno gostaria de pedir ajuda para realiza-la ou simplesmente pedir para fazer outra coisa obtendo a atenção do interlocutor (e.g., reforçamento positivo por atenção ou ação específica). Ou que, naquele momento, o aluno está motivado em produzir ou amenizar alguma estimulação sensorial no deu corpo (e.g., reforçamento automático), sabe-se que diversas dessas respostas podem ser incompatíveis com a tarefa de responder às demandas da avaliação ou de um contexto educacional de forma geral. No presente trabalho, somente as respostas que tinham a função de fuga/esquiva da tarefa e/u que eram incompatíveis com a continuidade do mesmo, fizeram parte da análise.

\section{CONSIDERAÇÕES FINAIS}


O TEA é um transtorno do desenvolvimento que possui características que podem levar a pessoa com este diagnóstico a enfrentar várias dificuldades no processo de aprendizagem. A literatura aponta que professores da rede regular de ensino têm dificuldade em saber quais conteúdos ensinar e quais procedimentos são mais adequados para o aluno com TEA (PAPIM; SANCHES, 2013). Uma ferramenta importante para direcionar o professor quanto ao que ensinar para o aluno com TEA é avaliação de repertório inicial (SKINNER; 1979; DE ROSE, 2004).

Os resultados do presente estudo, mostraram que os participantes obtiveram a classificação de 'Acerto Total' em poucas áreas do instrumento (4 do total de 13. A saber, A1 Esquema Corporal (P1 e P2), A8 Forma (P1 e P3) e A13 Coordenação Motora Fina (P1 e P3). Além disso, somente em uma área, a A4 Direção, os três participantes acertaram todas as tarefas propostas obtendo classificação AT. Esses resultados indicam que, embora todos os participantes tenham sido selecionados para o estudo justamente por já serem alfabetizados, e supostamente, já possuírem as habilidades prérequisito de alfabetização avaliadas pelo IAR, a apresentação do instrumento conforme proposto pelo manual não favoreceu que as crianças com TEA os exibissem respondendo com êxito a maioria das tarefas da avaliação.

$\mathrm{Na}$ contramão, apresentaram 'Muita dificuldade ou Alguma dificuldade' em realizar as tarefas da maioria das áreas avaliadas por este instrumento. Ainda que leiam, escrevam e sejam considerados alfabetizados.

A análise dos tipos de erros cometidos pelos participantes, aliada às informações de que todos já possuíam as habilidade pré-requisitos latestadas inclusive por protocolo de avaliação comportamental VBMAPP realizado em outro contexto pelos alunos), indicou que é possível que eles não tenham conseguido realizar as tarefas devido às instruções fornecidas e/ou às características do material de aplicação. Levantando assim a hipótese de que o "baixo" desempenho nas tarefas esteja mais relacionado ao material/contexto de avaliação do que à ausência dos repertórios avaliados. 
Dentre as características do IAR que podem estar relacionadas aos tipos de erros cometidos pelos participantes, destacam-se as seguintes: no caderno de resposta do aluno, são apresentadas várias tarefas por página; a maioria das respostas deve ser emitida por meio do comportamento de pintar e escrever (o que pode tornar o custo da resposta alto, por ser uma habilidade motora complexa para muitos alunos com TEA); as instruções para realização das tarefas são fornecidas por meio da fala, são variadas (por exemplo, em algumas tarefas a instrução é pintar, em outras é fazer uma bola (circular) e/ou fazer um $X$ em algum componente impresso na folha de respostas) e complexas, ou seja, requerem a execução de mais de uma ação ou ações que exigem o conhecimento de algum outro conceito além da própria ação (por exemplo, pintar a partir da escolha do lápis de uma cor específica) podendo assim, dificultar a compreensão da tarefa pelo aluno com TEA; alguns desenhos são ricos em detalhes, isso pode tornar-se um distrator para o aluno com TEA; e por fim, o manual não deixa claro quais estratégias utilizar para minimizar as possibilidades do aluno emitir comportamentos disruptivos relacionados às tarefas e 0 que 0 professor deve fazer diante deles, caso ocorram.

Outrossim, como mostram os dados, os participantes P1 e P2 emitiram alta ocorrência de comportamentos disruptivos durante a aplicação do IAR. P3, ainda que por menos tempo, também apresentou comportamentos disruptivos durante a avaliação. Tais comportamentos podem estar relacionados com a forma como as tarefas foram apresentadas.

Além disso, por meio de uma análise mais detalhada, pode-se perceber que os comportamentos de ameaça de agressão emitidos por Pl, ocorreram mais frequentemente durante a aplicação das tarefas de discriminação auditiva, onde a exposição às instruções orais era ainda maior.

Dado que a literatura tem demostrado a importância dos professores avaliarem as habilidades (BELISÁRIO, MATA \& CUNHA, 2008) e elaborarem propostas pedagógicas adaptadas ao estilo de aprendizagem da pessoa com TEA (CAMARGO \& BOSA, 2009), se faz necessário que os instrumentos de avaliação também considerem os padrões de comportamento comumente 
exibidos por indivíduos dessa população. Por exemplo, dificuldade de processar informações verbais e longas, possibilidade de responder sob controle restrito de estímulos (e.g., CALLOU; DE ASSIS, 2018; DA HORA; BENVENUTI, 2012), especialmente quando a estimulação visual é rica em detalhes e padrão de responder de forma restrita e rígida, podendo se estabelecer o controle inadvertido pela sequência de apresentação das atividades.

Considerando os resultados até o momento, um segundo estudo (cujo manuscrito está em fase de preparação para publicação futura) foi conduzido para avaliar o efeito de adaptações no instrumento original do IAR, na tentativa de ampliar os conhecimentos sobre sua aplicabilidade para indivíduos com TEA. Tal investigação foi ao encontro das propostas Capellini (2001) e Rabello et al. (2007), que também realizaram adaptações no IAR para avaliar o desempenho de alunos com deficiências e necessidades educacionais especiais. Futuros estudos das presentes autoras concentraram esforços em investigar o efeito de adaptações na apresentação das tarefas, instruções e procedimento de aplicação do IAR, sobre a realização da avaliação novamente com alunos com TEA.

Investigações futuras também poderiam se debruçar sobre o sistema de avaliação do desempenho do IAR que descreve critérios compatíveis com uma análise binária: se acerta $100 \%$ das respostas, entende-se que 0 aluno demonstrou a habilidade avaliada. Desempenhos menores de $100 \%$ de acerto, já são indicativos de 'Alguma dificuldade' e abaixo de 50\% de 'Muita dificuldade'. Importante considerar que o processo de ensino aprendizagem é dinâmico e que uma análise mais abrangente e menos dicotômica sobre o desempenho dos alunos, considerando a qualidade e a quantidade do erro e a quantidade de oportunidades apresentadas para avaliação podem ser críticas para o sucesso do ensino (DALYIII; MARTENS, 1997; MELO; HANNA; CARMO, 2014).

Embora o principal objetivo do trabalho tenha sido verificar se O IAR poderia ser aplicado em crianças com TEA para auxiliar no planejamento de professores da educação básica que descrevem ter muitas dificuldades 
sobre como atender essa população em contexto de ensino regular (PAPIM; SANCHES, 2013). Justamente por parecer ser uma ferramenta simples, rápida, cujas normativas, não requer conhecimentos prévios sobre as abordagens teóricas específicas ou as necessidades educacionais especiais e, portanto, poderia ser uma ferramenta muito útil para professores das primeiras séries de promoção de letramento e, sobretudo, para profissionais de AEE, Apoio Educacional Especializado. Ainda sim, reconhece-se a limitação de ter optado por um instrumento desenvolvido para crianças típicas e cuja forma de aplicação não contempla as particularidades de desenvolvimento das crianças com TEA.

Por fim, futuros estudos poderiam investigar se a possibilidade do instrumento, preferencialmente com adaptações que contemplem as características de aprendizado de pessoas com TEA, incluindo meios para favorecer habilidades básicas para seu letramento (GOMES, 2015), poderiam ser aplicados diretamente por professores de AEE da rede pública de ensino, favorecendo assim a possibilidade de ampliar o acesso a uma tecnologia útil aos contextos educação pública.

\section{REFERÊNCIAS}

AMERICAN PSYCHIATRIC ASSOCIATION. Diagnostic and Statistical Manual of Mental Disorder (5th ed.), 2013.

BELISÁRIO, J. MATA, O. CUNHA, P. A Inclusão Escolar de estudantes com autismo na rede municipal de educação de Belo Horizonte: Síntese da Frente de trabalho Autismo e Síndromes. Secretaria Municipal de Educação de Belo Horizonte, 2008. Disponível em:<

https://www.scielo.br/scielo.php?script=sci_arttext\&pid=s141365382010000300005>

BLANCO, R. A atenção à diversidade na sala de aula e as adaptações do currículo. In. COLL, C.; MARCHESI, A.; PALACIOS, J. A. (Orgs.).

Desenvolvimento psicológico e educação: transtornos de desenvolvimento e necessidades educativas especiais. Porto Alegre: Artmed Editora. 2004.

COOPER, J. O. et al. Applied behavior analysis. 2007. 
CALLOU, I. C.; DE ASSIS, G. J. A.; DE BORBA, M. M. C. Controle restrito de estímulos e autismo: Avaliação em tarefas de matching to sample com estímulos visuais. ACTA Comportamentalia, v. 26, n. 4, p. 417-431, 2018.

CAMARGO, S. H. BOSA, C. A. Competência social, inclusão escolar e autismo: um estudo de caso comparativo. Psic.: Teor. e Pesq., Brasília, v.28, n.3, p. 315-324, 2012. Disponível em <http://www.scielo.br/scielo.php?script=sci_arttext\&pid=\$01023772201200030 0007\&lng=en\&nrm=iso>. v28, n3, p:315-324. 2012.

CAPELLINI, V. L. M. F. A inclusão de alunos com necessidades educacionais especiais em classes comuns: avaliação do rendimento acadêmico. (Dissertação de Mestrado), Universidade Federal de São Carlos, São Carlos, SP, Brasil. 2001. Disponível em:<

https://www.academia.edu/24963419/a_inclus\%c3\%830_de_alunos_com_ne cessidades_educacionais_especiais_em_classes_comuns_avalia\%c3\%87\%c3 \%83o_do_rendimento_acad\%c3\%8amico>

DA HORA, C. L. et al. Controle inadvertido pela localização em tarefas de matching-to-sample com indivíduos com transtorno do espectro autista.

Revista Brasileira de Análise do Comportamento brazilian journal of behavior analysis. n.14, v1, p15-23, 2018. Disponível em:< https://periodicos.ufpa.br/index.php/rebac/article/view/7155/5538>

DALY III, E. J. et al. A model for conducting a functional analysis of academic performance problems. School Psychology Review, v. 26, n. 4, p. 554-574, 1997.

DA HORA, C. L.; BENVENUTI, M. F. L. Controle restrito em uma tarefa de matching-to-sample com palavras e sílabas: avaliação do desempenho de uma criança diagnosticada com autismo. Revista Brasileira de Análise do Comportamento, v. 3, n. 1, 2012.

DA HORA, C. L. et al. Controle inadvertido pela localização em tarefas de matching-to-sample com indivíduos com transtorno do espectro autista.

Revista Brasileira de Análise do Comportamento, v. 14, n. 1, 2018.

DE ROSE, J. C. Além da resposta correta: controle de estímulos e o raciocínio do aluno. In. HÜBNER, M. M. M.; MARINOTTO, M. (Orgs.). Análise do

Comportamento para a educação: contribuições recentes. Santo André: Esetec, 2004.

GLAT, R.; VIANNA, M. M.; REDIG, A. G. Plano Educacional Individualizado: uma estratégia a ser construída no processo de formação docente. Ciências Humanas e Sociais em Revista. Rio de Janeiro, v.34, n.12, p.79-100. 2012. Disponível em< http://www.ufrrj.br/SEER/index.php?journal=chsr\&page=article\&op=view\&pat $h \% 5 B \% 5 D=834>$ 
GOMES, C. G. S. Ensino de leitura para pessoas com autismo. Appris Editora e Livraria Eireli-ME, 2015.

GOMES, C. G. S., MENDES, E. G. Escolarização inclusiva de alunos com autismo na rede municipal de ensino de Belo Horizonte. Revista Brasileira de Educação Especia, Marília, v.16, n.3, p.375-396, 2010. Disponível em:< http://www.scielo.br/pdf/rbee/v16n3/v16n3a05. pdf. doi: 10.1590/S141365382010000300005>

LEITE, S.A.S. Instrumento de avaliação de repertório básico para a alfabetização. 3. Ed. 2013, São Paulo: EDICON. (Instrumento de avaliação)

LEITE, S.A.S. Instrumento de avaliação de repertório básico para a alfabetização. 3. Ed. 2013, São Paulo: EDICON. (Manual de aplicação)

LEITE, S.A.S. Preparando a alfabetização. $4^{a} e d .2015$. São Paulo: EDICON.

PAPIM, A. A. P. SANCHES, K. G. Autismo e inclusão: levantamento das dificuldades encontradas pelo professor do Atendimento Educacional Especializado em sua prática com crianças com Autismo (Monografia). Centro Universitário Católico Salesiano Auxilium, Lins, SP, Brasil, 2015.

PARTINGTON, J. W.; MUELLER, M. M. The Assessment of Basic Language and Learning Skill. Revised: The ABBLS-R. 2006.

PARTINGTON, J. W. MUELLER, M. M. The Assessment of Functional Living Skills: The AFLS-R. United States of America: Behavior Analysts, In and Stimulus Publications, 2013.

PEREIRA, M. E. M. MARINOTTI, M. LUNA, S. V. O compromisso do professor com a aprendizagem do aluno: contribuições da Análise do Comportamento. In. HÜBNER, M. M. C.; MARINOTTI, M. (Orgs.) (13 $3^{a}$ ed). Análise do Comportamento para a Educação: Contribuições recentes. Santo André, SP: ESETec. 2004.

PAPIM, A. A. P.; SANCHES, K. G. Autismo e Inclusão: Levantamento das dificuldades encontradas pelo professor no Atendimento Educacional Especializado em sua prática com crianças com autismo. 2013. 85 f. TCC (Graduação)-Curso de Psicologia, Centro Universitário Católico Salesiano Auxílium, Lins-Sp, 2013.

POPPOVIC, A.M. et al. Cartas as professoras de Alfa Um. São Paulo: Abril Educação, 1977.

MELO, R. M. de; HANNA, E. S.; CARMO, J. dos S. Ensino sem erro e aprendizagem de discriminação. Temas psicol., Ribeirão Preto, v. 22, n. 1, p. 207-222, abr. 2014. Disponível em:

<http://pepsic.bvsalud.org/scielo.php?script=sci_arttext\&pid=\$1413389X2014000100016\&lng=pt\&nrm=iso>. http://dx.doi.org/10.9788/TP2014.1-16. 
RABELLO, S.; MOTTI, T. F. G.; GASPARETTO, M. E. R. F. Avaliação educacional por meio do teste IAR em escolares com cegueira. Revista Brasileira de Educação Especial, Marília, v.13, n.2, p. 281-290, 2007. Disponível em:< http://www.scielo.br/pdf/rbee/v13n2/a09v13n2.pdf. doi: 10.1590/S141365382007000200009>

SANTIAGO, M. C. SANTOS, M. P. MELO, S. C. Inclusão em educação: processos de avaliação em questão. Ensaio: Avaliação e Políticas Públicas em Educação, v.25, n.96, p.632-651, 2017. Disponível em:

http://www.scielo.br/pdf/ensaio/v25n96/1809-4465-ensaio-S010440362017002500652.pdf. doi: 10.1590/s0104-40362017002500652

SCHOPLER, E. et al. Psychoeducational Profile Revised (PEP-R). Texas: Proed, 1990.

SKINNER, B. Tecnologia do Ensino. São Paulo: E.P.U. 1972.

SUNDBERG, M. L. The verbal behavior milestones assessment and placement program: VB-MAPP. Concord, CA: AVB Press. 2008.

Recebido em: 10 de setembro de 2020

Aprovado em: 06 de novembro de 2020

Publicado em: 10 de novembro de 2020 